\title{
Measurement of Velocity and Diameter of Individual Fertilizer Particles by an Optical Method
}

\author{
T. E. Grift; J. W. Hofstee \\ Department of Agricultural Engineering and Physics, Wageningen Agricultural University, Bomenweg 4, 6703 HD Wageningen, \\ The Netherlands \\ (Received 10 July 1995; accepted in revised form 26 October 1996)
}

Fertilizer spreaders are traditionally tested by using the collecting tray method. This method requires large indoor test facilities and is very expensive when applied on a large scale. A proposed alternative method is to measure the velocity and diameter of individual fertilizer particles emanating from the spreader and use a model to predict the landing spot of individual particles. The total spread pattern follows from accumulating a large number of particle landing spots. An optical device for measuring the velocity vector and dimensions of individual fertilizer particles was developed. The system proved capable of measuring the velocity of a $4 \mathrm{~mm}$ particle travelling at $29 \mathrm{~m} / \mathrm{s}$ within an accuracy of $5 \%$ and dimensions within $2 \%$. Two forms of the detector are discussed, namely, one and two dimensional versions respectively. (C) 1997 Silsoe Research Institute

\section{Notation}

$\Delta t_{f} \quad$ time difference between falling edges, $\mathrm{s}$

$\Delta t_{p} \quad$ pulse width from sensor array signal, $\mathrm{s}$

$\Delta t_{f x}$ time difference between falling edges in the $x$ direction, $\mathrm{s}$

$\Delta t_{p x} \quad$ pulse width from sensor array signal in the $x$ direction, $\mathrm{s}$

$\Delta t_{f x y} \quad$ time difference between falling edges from $x$ to $y$ axes, $\mathrm{s}$

$\Delta t_{f y} \quad$ time difference between falling edges in the $y$ direction, $\mathrm{s}$

$\Delta t_{p y} \quad$ pulse width from sensor array signal in the $y$ direction, $\mathrm{s}$

$v_{x}$ velocity in $x$ direction, $\mathrm{m} / \mathrm{s}$

$v_{y}$ velocity in $y$ direction, $\mathrm{m} / \mathrm{s}$

$D_{x} \quad$ dimension of particle in $x$ direction, $m$

$D_{y}$ dimension of particle in $y$ direction, $m$

$(x, y)$ passing position of particle, $(\mathrm{m}, \mathrm{m})$

\section{Introduction}

The Dutch government has announced an intention of requiring a periodic inspection of fertilizer spreaders used by farmers and/or contractors with respect to their spreading performance (Heestermans ${ }^{1}$ ). Proper testing of fertilizer spreaders requires large indoor test facilities to exclude environmental effects such as wind and rain. The traditional fertilizer spreader test uses the "collecting tray" method. A row of trays is placed perpendicular to the driving path of the tractor and after the spreading run, the mass collected in each tray is weighed. Since this procedure requires large investments when applied on a large scale, an alternative approach was proposed by Hofstee. ${ }^{2}$

The principle consists of a measurement device that moves around the spreader in a circular manner, at arbitrary heights, thus scanning the complete spreading zone. It has a small orifice $(3 \mathrm{~cm})$ whereby the velocity vector and diameter of particles passing through it are measured. These two variables are used in an aerodynamic model to predict the landing position of a particle. By accumulation of a large number of landing positions a complete spread pattern can be assembled.

\section{The optical sensor}

\subsection{Description}

The optical sensor detects particles by monitoring the signal of photo sensitive sensors. These devices have a "high" output when lit by a light source. By placing a large number of photo sensitive sensors in line (in this design 30), a sensor array is constructed. All individual photo sensitive sensors are logically connected in one OR function. In this way the signal 


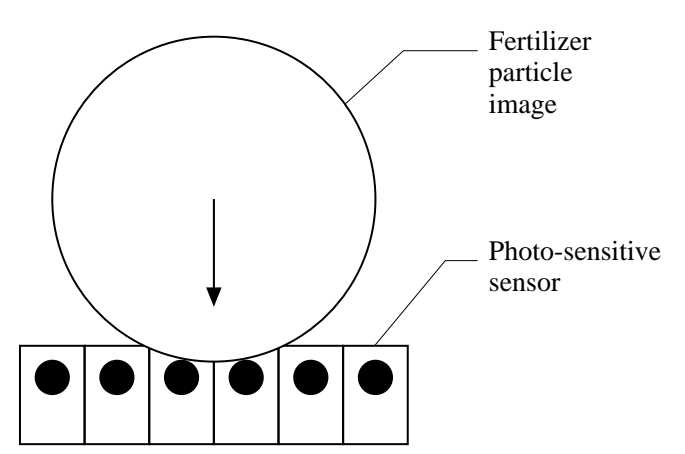

Fig. 1. Particle passing a sensor array

of the sensor array is "high" when all sensors receive light and "low" when any of the photo sensitive sensors is blocked by a particle, as illustrated in Fig. 1 .

A disadvantage of the sensor array is its physical dimensions. Each photo sensitive sensor has an active area in the shape of a circle with a diameter of about $5 \mathrm{~mm}$. This means that a fertilizer particle of $2 \mathrm{~mm}$ diameter could easily slip through two adjacent photo sensitive sensors and remain undetected. This problem is dealt with by using optical amplification. The principle is illustrated in Fig. 2. It shows a light source that produces a parallel beam, for which the conden- ser section of a slide projector is used. The light then passes first through a converging then a diverging lens, such that the image of a particle is amplified by a factor of eight.

The actual particle velocity and diameter measurement is done by monitoring the signals of two sensor arrays. Since the light paths being blocked are determined by the arrays of photo sensors, two light "layers" can be distinguished. From the time difference corresponding to a particle travelling from the upper layer to the lower, a velocity can be derived (assuming a perfectly perpendicular path), and from the time a particle blocks each sensor array, the diameter can be computed.

\subsection{One-dimensional measurement}

Figure 3 shows a spherical particle, passing the light sensors, together with the corresponding oscilloscope images. When a particle is travelling at a constant velocity perpendicular to the light beams the following equations apply.

Velocity

$$
v=\frac{b}{\Delta t_{f}}
$$

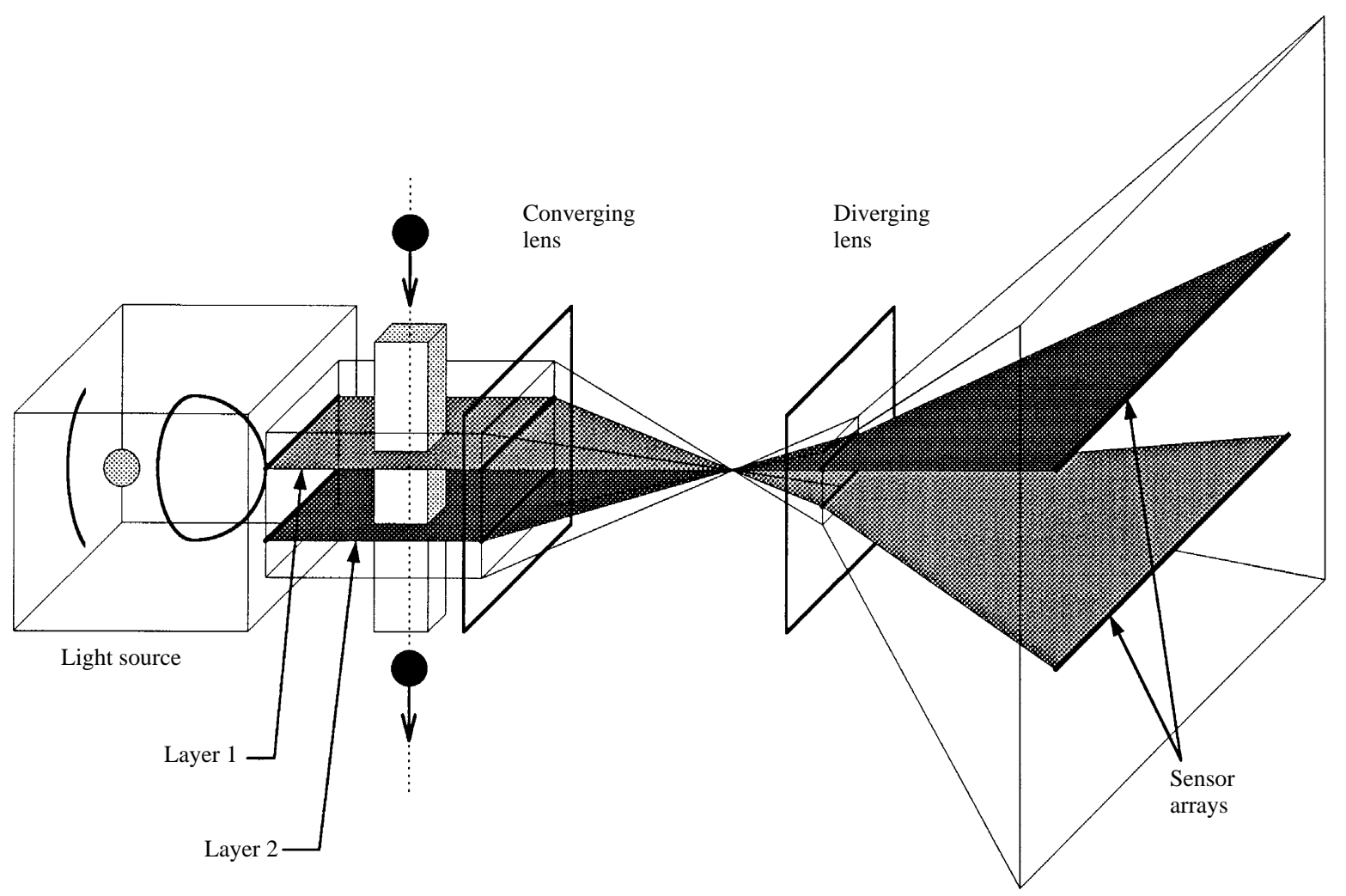

Fig. 2. Principle of the optical detector 

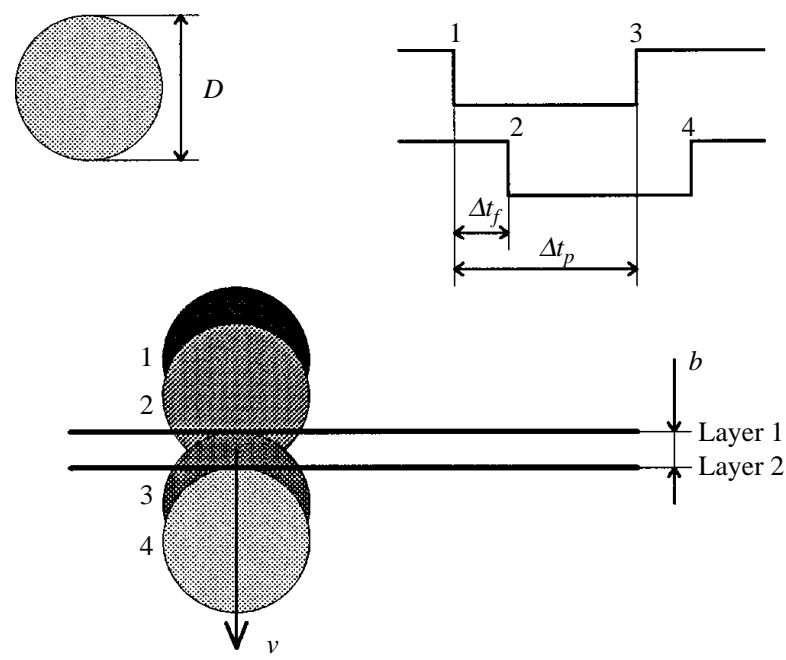

Fig. 3. One-dimensional particle detection

Diameter

$$
D=v \Delta t_{p}=b \frac{\Delta t_{p}}{\Delta t_{f}}
$$

The factor $b$ equals the distance between the sensor arrays $(27.0 \mathrm{~mm})$ divided by the amplification factor of eight. The "diameter" measured should be interpreted as one major dimension (under the assumption of no spin) of the particle and for spheres this is evidently equal to the diameter.

\subsection{Two-dimensional measurement}

The initial one-dimensional version of the detector was modified for two dimensional operation. The parallel layers were turned through $90 \mathrm{deg}$ by a mirror, such that particles blocked both layers twice. The two-dimensional construction also gives the advantage of determining the passing position of a particle. Again, the measured "diameters" have to be interpreted as two perpendicular principal axes. The principle is shown in Fig. 4 and the following equations apply.

Velocities

$$
v_{x}=\frac{b}{\Delta t_{f x}} \quad v_{y}=\frac{b}{\Delta t_{f y}}
$$

Diameters

$$
D_{x}=v_{x} \Delta t_{p x}=b\left(\frac{\Delta t_{p x}}{\Delta t_{f x}}\right) \quad D_{y}=v_{y} \Delta t_{p y}=b\left(\frac{\Delta t_{p y}}{\Delta t_{f y}}\right)
$$

Position

$$
x=v_{x} \Delta t_{x y}=b\left(\frac{\Delta t_{f x y}}{\Delta t_{f x}}\right) \quad y=v_{y} \Delta t_{f x y}=b\left(\frac{\Delta t_{f x y}}{\Delta t_{f y}}\right)
$$

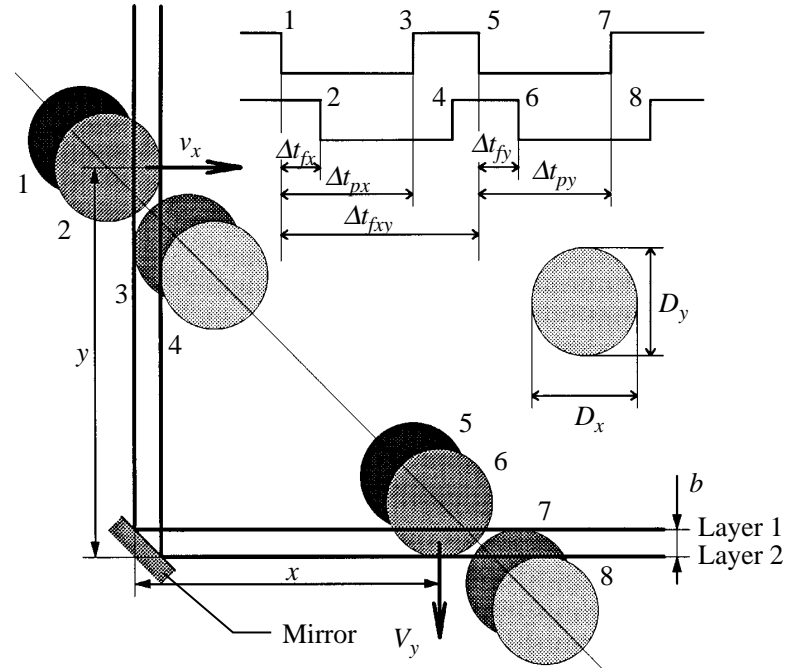

Fig. 4. Two-dimensional particle detection

\section{Results}

\subsection{One-dimensional measurements}

As a test object a pellet was fired with a $5.5 \mathrm{~mm}$ air gun. Figure 5 shows the pulses of both sensor arrays. The values of $\Delta t_{f}$ and $\Delta t_{p}$ were measured and found to be $26.50 \mu$ s and $44.55 \mu$ s respectively. By using Eqns (1) and (2) the velocity was found to be $127 \mathrm{~m} / \mathrm{s}$, and the vertical dimension of the pellet $5.67 \mathrm{~mm}$. The real length of the pellet was also measured with a micrometer and found to be $5.60 \mathrm{~mm}$. Hence the accuracy

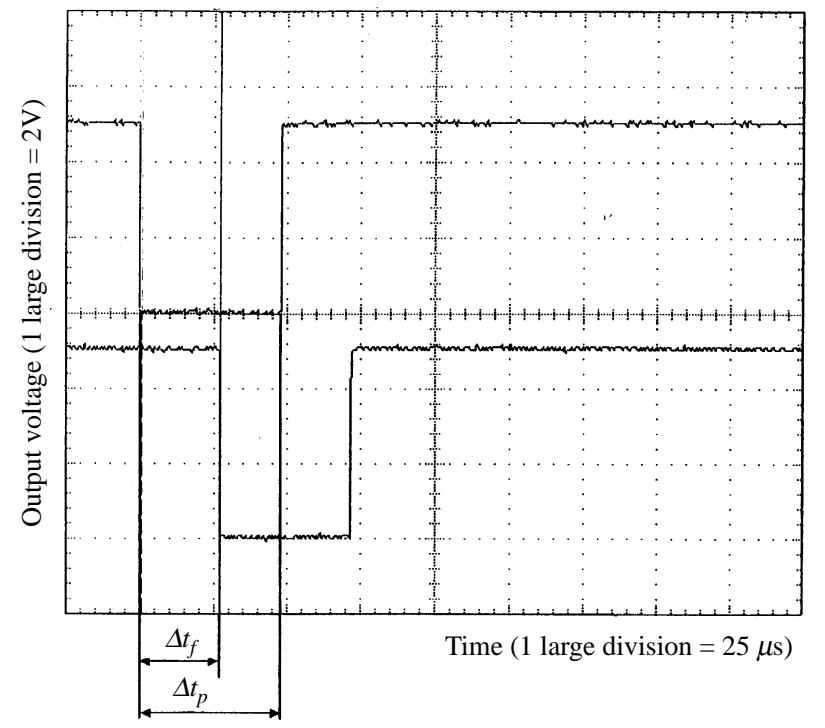

Fig. 5. Oscilloscope image of pellet passing at $125 \mathrm{~m} / \mathrm{s}$ (one-dimensional case) 
of the dimension measurement was within $2 \%$. The accuracy of the velocity measurement could not be determined, since the real velocity of the pellet was unknown.

\subsection{Two-dimensional measurements}

For testing, a $4 \mathrm{~mm}$ plastic particle was launched with a special device, a particle accelerator described by Grift and Hofstee. ${ }^{3}$ The estimated initial velocity of the particle was derived from the rotational velocity of the particle accelerator disc, $(\cong 29 \mathrm{~m} / \mathrm{s})$. Figure 6 shows the output signals of both sensor arrays.

The time differences were measured and found to be

$$
\begin{aligned}
\Delta t_{f x} & =165 \mu \mathrm{s} \\
\Delta t_{p x} & =195 \mu \mathrm{s} \\
\Delta t_{f x y} & =575 \mu \mathrm{s} \\
\Delta t_{f y} & =180 \mu \mathrm{s} \\
\Delta t_{p y} & =210 \mu \mathrm{s}
\end{aligned}
$$

Substitution in Eqns (3)-(5) resulted in $v_{x}=$

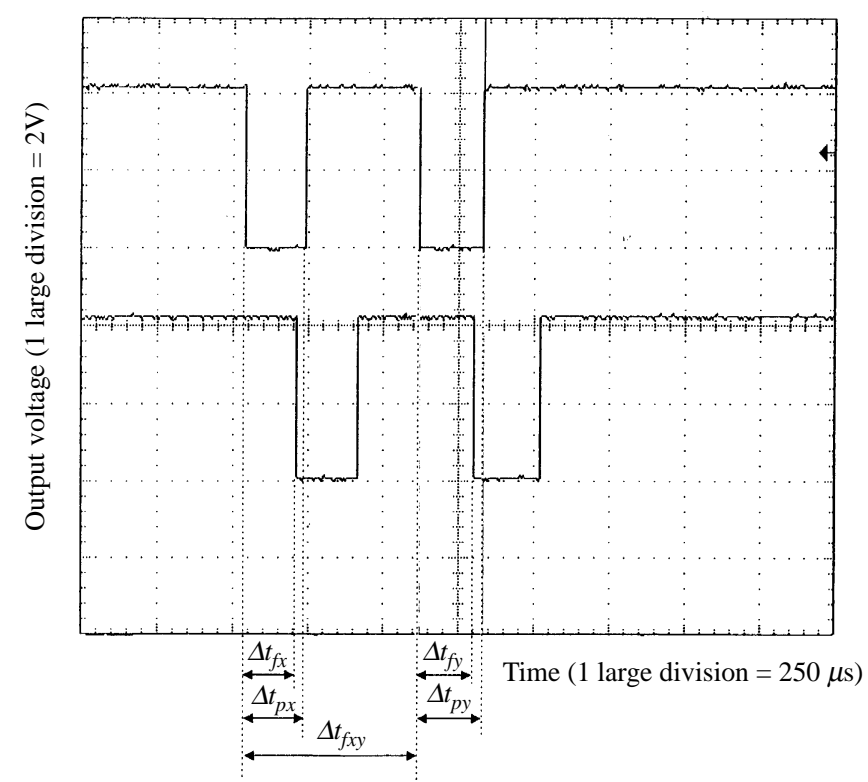

Fig. 6. Oscilloscope image of plastic sphere passing perpendicular light beams (two-dimensional case)
$20.45 \mathrm{~m} / \mathrm{s}$ and $v_{y}=18.75 \mathrm{~m} / \mathrm{s}$ for velocities and $D_{x}=$ $3.99 \mathrm{~mm}$ and $D_{y}=3.94 \mathrm{~mm}$ for diameters respectively. The resultant velocity was calculated by combining the perpendicular velocity vectors $v_{x}$ and $v_{y}$, and found to be $27.75 \mathrm{~m} / \mathrm{s}$. The errors in the diameter and velocity measurement were within 2 and 5 percent respectively. The position measurement $(x, y)$ was $x=$ $11.8 \mathrm{~mm}$ and $y=10.8 \mathrm{~mm}$ respectively.

\section{Conclusions}

The sensor was capable of on-line determination of the velocity and simultaneous measurement of the dimensions of a fertilizer particle. A test of the one-dimensional version showed that the system could estimate the length of an air gun pellet to within $2 \%$ when the pellet was travelling at approximately $125 \mathrm{~m} / \mathrm{s}$. The dimensional version was tested by launching a plastic sphere with a known initial velocity and diameter. Here the diameter was measured within an accuracy of $2 \%$ and the velocity within $5 \%$. The test results show that the photo-interrupter approach is capable of detecting particle size and velocity but there are various problems to be solved in order to apply the method to testing of fertilizer spreaders. The system showed acceptable accuracy and can be built from low cost, standard components.

\section{References}

${ }^{1}$ Heestermans J M A J Concept beleidsplan voor de verplichte onderhoudskeuring van kunstmeststrooiers, Rapport van de Initiatiefgroep Keuring Kunstmeststrooiers (Concept policy plan for mandatory maintenance calibration of fertilizer spreaders, Initiative group report for calibration of fertilizer spreaders), 01-08-1993

${ }^{2}$ Hofstee J W Handling and spreading of fertilizers: Part 3, Measurement of particle velocities and directions with ultrasonic transducers, theory, measurement system and experimental arrangements. Journal of Agricultural Engineering Research, 1994, 58(1), 1-16

${ }^{3}$ Grift T E; Hofstee J W Development of a Fertilizer Particle Accelerator, Journal of Agricultural Engineering Research, 1997, 66, 229-233 